\title{
Archives
}

\section{Les prochains défis de la Division de la gestion de documents et des archives de l'Université de Montréal}

\section{DIANE BAILLARGEON}

Volume 46, numéro 2, 2017

$50^{\mathrm{e}}$ anniversaire de la DGDA

URI : https://id.erudit.org/iderudit/1040389ar

DOI : https://doi.org/10.7202/1040389ar

Aller au sommaire du numéro

Éditeur(s)

Association des archivistes du Québec (AAQ)

ISSN

0044-9423 (imprimé)

2369-9256 (numérique)

Découvrir la revue

Citer cet article

Baillargeon, D. (2017). Les prochains défis de la Division de la gestion de documents et des archives de l'Université de Montréal. Archives, 46(2),

163-175. https://doi.org/10.7202/1040389ar
Résumé de l'article

Depuis le début des années 1970, la Division de la gestion de documents et des archives de l'université de Montréal s'est imposée par son innovation, sa rigueur et son désir de communiquer son savoir et son savoir-faire. Reconnue internationalement pour l'exemplarité de ses méthodes de travail, l'article fait un retour sur les principales réalisations de la Division pour ensuite exposer les défis qui l'occuperont en terme de gouvernance informationnelle, de gestion des archives numériques et de valorisation des archives patrimoniales. L'auteure termine l'article avec les nouvelles définitions de la mission, de la vision et des valeurs de la Division ainsi qu'en soulignant l'importance d'entreprendre un exercice d'évaluation et de mesure de leurs services auprès de leurs partenaires. 


\section{0 $0^{\mathrm{E}}$ ANNIVERSAIRE DE LA DGDA}

\section{Les prochains défis de la Division de la gestion de documents et des archives de l'Université de Montréal}

\section{DIANE BAILLARGEON}

Directrice, Division de la gestion de documents et des archives, Université de Montréal.

\section{BREFS RAPPELS DES GRANDES RÉALISATIONS DE LA DGDA}

Ce colloque a permis de mettre en évidence le chemin parcouru par la Division de la gestion de documents et des archives de l'Université de Montréal depuis sa création le 14 juillet 1966 jusqu'à aujourd'hui. De modeste service dont le mandat était limité au greffe de la direction, comptant un seul employé pour seconder l'Archiviste universitaire et logé dans des locaux inadéquats, la DGDA est devenue ce qu'elle est aujourd'hui: une division bien structurée comptant onze employés qualifiés et compétents avec un rattachement organisationnel bien établi et des programmes bien pensés, une unité qui recueille l'adhésion de la direction et des unités et, surtout, un service qui a une excellente réputation tant au sein de I'Université que dans le milieu archivistique québécois, canadien et même international. 
Lors de ce colloque, les présentations des différents intervenants ont démontré de manière éloquente la matière et la manière, deux vocables empruntés à mon prédécesseur Claude Minotto, qui ont permis à la DGDA de se démarquer très tôt dans le paysage archivistique québécois. Les conférenciers de la journée ont évoqué tantôt l'une et tantôt l'autre de ces caractéristiques. Permettez-moi pour ma part de m'attarder tout d'abord à la manière, en rappelant certaines valeurs qui continuent de définir ce que nous sommes aujourd'hui et qui nous guideront encore longtemps dans l'élaboration et l'atteinte de nos prochains défis. Et je parle ici de la détermination, de l'innovation et de la générosité.

En premier lieu, la détermination. II en a fallu aux différents directeurs de la DGDA pour obtenir les ressources nécessaires à l'atteinte de leurs objectifs. Ils ont dû établir un diagnostic, proposer des solutions, démontrer la nécessité d'obtenir ce dont ils avaient besoin, demander, demander encore, malgré les cycles de restrictions budgétaires qui nous semblent se rapprocher sans cesse, afin d'obtenir ce qui est essentiel pour la gestion des archives d'une grande université.

Dès le départ, cette détermination s'est fondée sur la conviction acquise par le savoir. Tout au long des 50 dernières années, la Division a eu la chance d'être dirigée et de pouvoir compter sur des archivistes qui s'abreuvaient aux meilleures sources d'information disponibles à leur époque. Avant que ne soit dispensée une formation universitaire spécifique à l'archivistique, les premiers directeurs ont acquis leurs connaissances en effectuant des stages aux Archives nationales des États-Unis à Washington, à la Direction des Archives de France ou aux Archives publiques du Canada. Avant la tenue des premiers congrès de I'Association des archivistes du Québec (AAQ), ils fréquentaient déjà ceux de la Society of American Archivists ou de l'Americain Records Managers Association (ARMA) ou participaient aux congrès et tables rondes organisés par le Conseil international des archives (CIA). Leur formation était aussi faite de contacts avec d'autres professionnels regroupés au sein de comités de toute nature et par la lecture d'ouvrages et d'articles scientifiques. Tout était bon pour voir ce qui se faisait de meilleur et de l'importer ici, à l'Université de Montréal.

Finalement, cette détermination s'est appuyée sur le soutien des différents secrétaires généraux qui dès la création du Service ont été les officiers de qui relevait la direction de la DGDA. Ces derniers ont cru en 
la nécessité de créer un Service des archives et ont mis tout leur poids pour défendre les programmes mis en place pour atteindre les objectifs fixés par la direction.

La détermination des différents agents a permis à la DGDA d'innover de mille manières. Du dépôt, en 1969, d'un mémoire présenté à la Commission conjointe du Conseil et de l'Assemblée universitaire par Luc-André Biron qui a profondément modifié le mandat et la place de la DGDA à l'Université, à la création du premier site Internet de la Division en 1999, à l'implantation de SyGED en 2007, à la mise en ligne des nouvelles règles de gestion à l'automne 2015, la DGDA n'a eu de cesse d'explorer de nouvelles manières de remplir son mandat.

L'innovation nous a aussi permis de suivre l'évolution technologique passant, en 2007, de l'atelier de microfilmage, implanté au début des années 1970, à la numérisation sérielle de masse, puis à la gestion des archives nées numériques, qu'elles soient structurées ou non structurées.

L'innovation nous a aussi amenés à modifier nos façons de faire, ce qui nous a permis de redéployer nos ressources humaines pour continuer de répondre aux besoins changeants de I'Université. C'est cet esprit d'innovation qui nous a fait plonger dans la refonte de notre Système officiel de classification avec ses 39 catégories fondées sur des sujets, vers une structure allégée de 12 séries basées sur les grandes fonctions de l'Université.

C'est encore ce désir d'innover qui nous fait prendre le virage vers le logiciel de gestion intégré de documents SyGED, faisant de I'Université de Montréal, un précurseur dans la mise en place d'un tel système. Être à l'avant-garde comporte son lot d'insécurité, puisque nous ne pouvons pas nous tourner vers des collègues pour trouver des solutions aux problèmes qui se posent inévitablement. Mais le pari a été gagné. À ce jour, 44 unités déposent des documents dans SyGED et 270 personnes I'utilisent régulièrement.

L'esprit d'innovation nous a aussi fait prendre le virage du Web 2.0, une des premières sinon la première université à se lancer dans les réseaux sociaux au Québec. Nous avons aussi accueilli un «artiste en résidence » qui nous a préparé l'affiche que nous avons remise à chaque conférencier en guise de remerciement en plus de préparer quelques PowerPoint qui peuvent servir d'écran de veille. 
Finalement, ce désir d'innover nous a amenés à plonger dans la gouvernance informationnelle, autre secteur où nous faisons office de précurseur dans le monde universitaire. Sans trop de préparation, mais avec détermination et pragmatisme, nous nous sommes lancés dans cette opération dont les retombées positives ne cessent de nous étonner. Cette incursion dans la gouvernance informationnelle est d'ailleurs à la base du volet sécurité de nos règles de gestion et sur la mise en place du projet de stockage des documents des unités dont il est fait mention dans le texte de Taïk Bourhis et Nathalie Denis que vous trouverez dans ce numéro.

Alliée à la détermination et à l'innovation, la générosité définit bien la DGDA. C'est la générosité de ses membres qui est à l'origine de leur implication bénévole dans le milieu archivistique autant à l'AAQ qu'au Réseau des archives du Québec (RAQ) ou au Conseil canadien des archives (CCA), mais aussi dans les Campagnes des paniers de Noël. C'est ce même esprit qui nous amène à accepter plusieurs stagiaires chaque année. Des stagiaires de I'EBSI, bien sûr, mais pas uniquement. Nous recevons aussi régulièrement des stagiaires des programmes de technique de la documentation, dont une est avec nous aujourd'hui ou encore des stagiaires internationaux francophones venus de France, de Suisse, de Belgique ou d'ailleurs.

S'ils nous offrent une prestation de travail, ces stagiaires sont en contrepartie accueillis au sein de notre équipe et chacun a la chance de rencontrer tous les membres de la Division afin d'avoir une image complète de notre mandat et de notre manière de le remplir.

C'est encore par générosité que la DGDA partage ses innovations et ses outils de gestion via le site Internet, plutôt que de les réserver aux employés de l'Université et de ne jamais refuser de partager son savoir avec d'autres services d'archives par le biais de conversations téléphoniques, de rencontres informelles ou de démonstrations.

Plusieurs des membres du personnel de la DGDA ont aussi été chargés de cours ou conférenciers dans différents établissements, particulièrement l'École de bibliothéconomie et des sciences de l'information, autre façon de partager leur savoir avec générosité.

Finalement, je veux souligner la générosité des membres de la DGDA et de ses amis, dont les dons permettront, au cours du cocktail qui suivra 
ce Colloque, de remettre les premières bourses Michel Champagne-DGDA pour la formation en archivistique.

\section{LA GOUVERNANCE INFORMATIONNELLE}

Comme nous venons de le voir, au fil de ses 50 ans d'existence, la DGDA n'a jamais eu peur d'innover. De l'exploration de «l'amont » dans les années 1970 à l'implantation de SyGED au milieu des années 2000, nous avons souvent été parmi les pionniers et nous n'avons jamais été avares de nos connaissances et de nos expériences en ces domaines.

Cette tradition se poursuit en ce qui concerne le vaste champ de la gouvernance informationnelle. Déjà, notre université est une des premières, sinon la première à avoir complété la catégorisation de ses actifs informationnels. Cet exercice, qui est devenu un passage obligé depuis l'adoption de la Loi sur la gouvernance et la gestion des ressources informationnelles des organismes publics et des entreprises du gouvernement (R.L.R.Q., chap. G-103), a permis à la DGDA d'explorer une avenue nouvelle. Cette incursion du côté de la sécurité de l'information et de la gestion des risques informationnels constitue une occasion favorable de nous positionner stratégiquement au sein de I'Université, occasion que nous avons saisie avec enthousiasme.

Si nous laissons à nos collègues des technologies de l'information le soin de concevoir les outils permettant d'assurer la disponibilité des réseaux informatiques et de télécommunication, notre expertise en ce qui concerne le contenu et la valeur de l'information produite par les unités nous donne une longueur d'avance en ce qui concerne les mesures à mettre en place pour assurer l'intégrité et la confidentialité de l'information tout au long de son cycle de vie.

Le premier exercice de catégorisation est maintenant terminé et nous en sommes à faire le bilan de l'exercice. Un des prochains défis de la DGDA est donc de s'impliquer dans l'évaluation des pratiques documentaires dans les unités afin d'évaluer les risques que peut causer à l'institution ce que Dominique Maurel, dans ses recherches sur la gouvernance informationnelle, a identifié comme étant un "ordre informationnel négocié » né de la «diversité des pratiques informationnelles (institutionnelles, de groupe et individuelles) adoptées 
pour gérer les documents d'activités [...et ] qui fragilisent les efforts de gouvernance informationnelle» (Maurel, 2013).

II ne s'agit pas tant là d'un nouveau territoire à découvrir que d'une nouvelle façon de définir les paramètres de notre mandat et de nos actions, de repositionner nos services et nos programmes dans une perspective de gouvernance et de sécurité de l'information et de démontrer notre apport singulier et incontournable à cette nouvelle exigence à la fois législative et stratégique pour l'institution.

Depuis plusieurs années déjà, nous avons établi des liens étroits avec la Direction des technologies de l'information et de la communication (DGTIC), de tels liens se tissent maintenant avec le Bureau de la vérification interne (BVI) et la Direction de la performance organisationnelle et la gestion des risques. Ces alliances stratégiques ne peuvent qu'être bénéfiques à notre positionnement et à l'efficacité de notre action.

Par exemple, la DGDA a participé à des ateliers d'évaluation des risques de projets institutionnels ayant une incidence sur la sécurité de I'information aux côtés de la DGTIC. Ce genre de collaboration doit non seulement se poursuivre, mais se multiplier afin que l'étendue de notre champ d'expertise soit connue et valorisée.

À cet égard, la mise en ligne des règles de gestion qui intègrent, dans une facture simplifiée, le contenu de notre Système officiel de classification (SOC) et de notre calendrier de conservation auquel nous avons ajouté des indications touchant à la sécurité de l'information, est un exemple frappant. Outre sa facilité d'utilisation, le but de ce nouvel outil est aussi de montrer notre implication dans la sécurité de l'information. Pour I'instant limitées aux seuls documents papier, ces règles présenteront aussi prochainement des directives pour le stockage des fichiers numériques non structurés. Tout un défi à relever.

\section{LA GESTION DES ARCHIVES NUMÉRIQUES}

II n'y a rien d'original à dire que la gestion des archives numériques constitue un des plus grands défis auxquels nous aurons à faire face. On parlait déjà du «bureau sans papier» dans les années 1980. À l'époque cela était presque vu comme une utopie, comme d'envoyer un humain sur Mars. Mais le temps a passé et de plus en plus de processus 
d'affaires sont aujourd'hui automatisés: les admissions se font en ligne et les documents en appui à la demande sont numérisés à la source, de plus en plus de transactions se font en ligne dans des systèmes centraux, sans parler de la prolifération de wikis pour le dépôt des documents de réunions de diverses instances. Bien sûr, plus d'un millier de boîtes sont encore transférées aux archives chaque année, mais le volume diminue et plusieurs unités impriment de moins en moins de documents.

Dans ces circonstances, le soutien aux unités pour la gestion de leurs fichiers non structurés est une criante nécessité. II n'y a qu'à constater les nombreux appels faits à notre Division pour venir en aide aux unités et l'accueil plus que favorable fait à nos interventions pour structurer les espaces de stockage dans les facultés, pour voir que le fruit est mûr pour intégrer la gouvernance documentaire.

Les utilisateurs, jusqu'à très récemment jaloux de leur autonomie dans l'organisation de leurs documents, réalisent que sans une organisation rigoureuse et rationnelle de leur information, ils ne s'y retrouvent plus. Le projet de stockage et de partage de fichiers arrive donc à point nommé pour répondre à ce besoin. Implanter une structure de répertoire personnalisée basée sur le SOC dans près de deux cents unités demeure cependant un défi auquel nous nous préparons à faire face. Cela se fait autant par l'élaboration d'un programme de formation adapté, que par la préparation d'outils de gestion pour soutenir les unités, ainsi que par une collaboration étroite avec la DGTIC en tout ce qui concerne les aspects technologiques.

II en est de même pour la gestion des courriels, que ce soit pour la gestion des dossiers étudiants durant toute la durée de leur parcours académique ou pour soulager le personnel des décanats, des solutions doivent être pensées pour soutenir les différents intervenants. Trop longtemps laissée à l'initiative de chaque individu, l'organisation de l'information reçue ou transmise par courriel est cruciale dans les processus de travail. Nous ne pouvons donc plus laisser ce pan de l'information à l'écart de nos programmes de gestion. Des pistes de solutions sont déjà connues et les formations sur la gestion des courriels remportent des succès fulgurants. Mais, le problème demeurera tant que la mise en application reposera sur le bon vouloir des individus impliqués.

Alors que les fichiers non structurés et les courriels demandent la collaboration de chacun, la gestion des données issues des systèmes 
centraux pose un défi différent, mais tout aussi exigeant. D’une part, les règles de conservation à cet égard sont encore trop calquées sur I'univers analogique et, d'autre part, les systèmes n'ont pas tous intégré des mécanismes d'élagage de l'information. Soit parce que les archivistes n'ont pas été conviés à leur élaboration, ou parce qu'au moment de leur conception, cette question était vue comme relevant d'un futur trop éloigné pour qu'on $s^{\prime} y$ intéresse, la question de la gestion des données reste un territoire à conquérir pour les prochaines années. En plus de la question de l'élagage, il faudra aussi bientôt se pencher sur celle de la lisibilité et de l'intelligibilité des données que nous voulons conserver. Dans ce secteur aussi, il nous faudra faire équipe avec les spécialistes des technologies de l'information pour faire face à ce défi.

Si certains projets sont encore à l'étape embryonnaire, d'autres sont déjà en marche, tel le chantier du dossier étudiant électronique. En ce moment hybride et éclaté, l'Université est à définir les contours que devrait prendre un dossier étudiant qui répondrait aux besoins de tous les intervenants tout en assurant la disponibilité, la fiabilité et la confidentialité de l'information qui y est contenue. La DGDA, en tant que gardienne de l'intégrité des documents et de l'application de la règle de conservation du dossier étudiant, est partie prenante à ce projet majeur et structurant pour l'Université.

\section{LA VALORISATION DES ARCHIVES PATRIMONIALES}

Nous nous sommes attardés jusqu'à présent aux défis découlant de la gestion des archives courantes institutionnelles. Mais notre mandat englobe aussi la gestion des archives patrimoniales, qu'elles soient institutionnelles ou de source privée. Dans ce domaine également, les défis à relever sont importants et stimulants.

Au premier chef, il faut parler de l'accessibilité de ces archives. Les conserver c'est bien, mais l'objectif ultime est de les rendre accessibles et de les mettre en valeur, dans le respect des lois et conventions applicables. Si nous avons acquis un système de gestion intégrée de documents qui sert aussi de dépôt numérique fiable, SyGED n'est pas destiné à la diffusion des archives patrimoniales. Une fois versés ou acquis, une fois décrits et numérisés, il reste encore à faire connaître ces documents.

Depuis quelques années déjà, nous avons fait notre entrée sur les réseaux sociaux et avons mis en ligne près de 800 photographies sur Flickr et plus de 
3500 documents iconographiques sont accessibles à partir de la plateforme de diffusion Calypso. Mais, cela reste une goutte d'eau comparativement à la totalité de nos ressources. Bien sûr, notre site Internet présente le catalogue des fonds d'archives, dont les notices descriptives sont repérables par les moteurs de recherches, mais bien d'autres choses encore pourraient être faites pour faciliter la recherche à partir de n'importe quel ordinateur. La Ville de Montréal et I'Université Concordia, pour ne nommer que ces institutions-là, ont adhéré à la plateforme ICA-ATOM, la même qui est utilisée par ARCHIVESCANADA.CA pour mettre en ligne les descriptions et les documents numérisés. Le besoin est là, la technologie existe, il ne reste qu'à y consacrer les ressources nécessaires pour concrétiser ce rêve d'une plus grande accessibilité.

Cela implique aussi de mettre les efforts pour accroître la numérisation de nos documents, tant iconographiques qu'audiovisuels. Dans ce dernier cas, cela permettrait aussi d'en assurer la pérennité, car les appareils de lecture des supports analogiques se font de plus en plus rares. Mais, les ressources sont minces pour faire face à ce grand défi. II faut donc examiner des solutions innovantes, comme la mise sur pied de partenariats externes pour assurer la commercialisation d'une partie du patrimoine pour en assurer la préservation. Voilà, le mot est lâché! D'autres, comme $B A n Q, s^{\prime} y$ sont frottés et les résultats semblent satisfaisants. II faut, bien entendu, évaluer le potentiel des collections, trouver les partenaires possibles, établir des stratégies de négociation qui permettront à chaque partenaire d'en sortir gagnant. C'est en soi tout un programme et tout un défi, auquel il faudra bien faire face si nous voulons être en mesure d'assurer la pérennité et l'accessibilité de nos collections.

\section{MISSION, VISION, VALEURS}

Au terme de 50 années de travail, et après une journée à regarder le chemin parcouru, le temps est venu de définir ce vers quoi nous voulons aller. S'appuyant sur des assises solides, la DGDA a décidé de se doter d'un document porteur identifiant sa mission, sa vision et ses valeurs pour les années à venir.

Le contenu de ce document est le fruit d'une réflexion faite en équipe et discuté avec le secrétaire général; il représente donc bien la DGDA aujourd'hui. 


\subsection{La mission}

La définition actuelle de notre mission relève plutôt de la description de son étendue. II y est précisé que le mandat de la Division couvre les trois âges des documents et qu'il vise aussi bien les archives institutionnelles que celles acquises de source privée.

Cette affirmation avait sa raison d'être au moment où l'archivistique intégrée était encore à faire ses marques. De plus, il était important de bien faire comprendre aux unités que la notion d'archives ne se limite pas aux documents à conservation permanente. Le concept d'archives - à la fois numérique et analogique, regroupant les documents de leur création à leur élimination ou leur conservation permanente - est maintenant bien connu dans le milieu archivistique et les programmes et les services que rend la DGDA en matière d'archives courantes étant de plus en plus utilisés, il est possible de choisir plutôt une formulation axée vers les objectifs à atteindre.

C'est aussi avec fierté que je vous présente la nouvelle mission de la DGDA:

Mettre en place une gouvernance documentaire exemplaire afin de:

Contribuer à la transparence administrative et à la défense des droits de l'Université.

Assurer l'accès à l'information nécessaire à l'atteinte des objectifs des membres de la communauté universitaire en temps utile et dans le respect des lois et conventions applicables.

Accompagner les unités académiques et administratives dans la réalisation de leur mandat par une meilleure gestion de leur information.

Constituer, préserver et valoriser la mémoire de l'Université de Montréal.

Renforcer le sentiment d'appartenance des membres de la communauté universitaire.

Exercer un leadership en matière archivistique et en faire bénéficier le milieu par le partage d'expertise. (Université de Montréal. Division de la gestion de documents et des archives, 2016) 
La nouvelle mission a l'avantage d'être davantage liée aux grandes orientations puisqu'elle positionne la DGDA comme une unité de soutien aux membres de la communauté universitaire pour atteindre leurs objectifs liés à l'enseignement et à la recherche. La mission renforce aussi le rôle de la DGDA dans la création, I'organisation, I'utilisation et la disposition des documents numériques et la sécurité de l'information, tout en soulignant la fonction fédérative des archives patrimoniales. La mission met aussi en évidence le souci, maintes fois souligné durant ce colloque, qu'a eu la DGDA tout au long de son existence, de partager son savoir et son savoir-faire.

\subsection{La vision}

La mission s'accompagnera dorénavant d'une vision. Alors que la mission définit ce que l'on fait, la vision permet de déterminer comment celle-ci sera réalisée. La vision donne un élan vers l'avenir, nous amène à nous projeter vers un idéal à atteindre. La vision de la DGDA se décline ainsi:

Mettre à la disposition des unités académiques et administratives des programmes et des services efficaces et fiables permettant aux membres de la communauté universitaire d'améliorer leur gouvernance documentaire. Augmenter la visibilité et la notoriété de la DGDA afin que les unités ainsi que les chercheurs connaissent et utilisent encore plus nos programmes et nos services. Partager nos connaissances et notre savoir-faire avec le milieu archivistique. (Université de Montréal. Division de la gestion de documents et des archives, 2016)

La vision met en évidence les qualités que doivent posséder nos programmes et nos services afin d'être en mesure de bien remplir notre mission. L'efficacité et la fiabilité sont deux maîtres-mots en matière de gestion des archives, particulièrement lorsqu'il est question d'archives numériques ou électroniques. Les programmes actuels qui vous ont été exposés par Taïk Bourhis, Nathalie Denis et Michel Champagne témoignent de l'importance de plus en plus grande accordée à nous faire connaître, autant à l'interne qu'à l'externe. Finalement, la transmission de notre expertise et le partage d'information continuent de faire partie de notre ADN et il nous semblait important de l'intégrer dans notre vision. 


\subsection{Les valeurs}

En plus de s'être donné une mission renouvelée et une toute nouvelle vision, la DGDA a défini des valeurs sur lesquelles elle s'appuiera pour remplir son rôle. Parmi toutes celles qui s'offraient à nous, notre choix s'est arrêté sur les suivantes:

- Rigueur et fiabilité

- Service à la clientèle

- Souci de l'accessibilité des archives dans le respect de la confidentialité

- Communication efficace

- Leadership et partage de notre expertise (Université de Montréal. Division de la gestion de documents et des archives, 2016)

Si la rigueur et la fiabilité sont essentielles pour que les membres de la communauté universitaire continuent de nous confier leurs archives en toute confiance, le souci du service à la clientèle y est aussi pour beaucoup. À ce chapitre, nous mettons un point d'honneur à répondre rapidement et efficacement aux demandes qui nous sont faites.

La pluralité de nos interlocuteurs et la diversité des archives qui nous sont confiées nécessitent que nous nous préoccupions tout à la fois de la confidentialité et de l'accessibilité des archives. Les règles de gestion, les procédures liées aux rappels des documents de même le respect des restrictions d'accès négociées au moment de la cession des fonds en sont quelques illustrations.

La clarté de nos communications, tant dans nos formations de groupes, dans les systèmes et outils accessibles sur notre site Internet que dans la réponse aux demandes des chercheurs constitue une condition sine qua non de l'application de nos programmes. C'est cette recherche de clarté qui nous pousse à améliorer sans cesse nos outils de gestion, nos aidemémoire et nos procédures, car la clarté des communications commence entre les membres de la Division avant de s'étendre à l'ensemble de la communauté universitaire.

Déjà présent dans la mission et la vision, le partage de notre expertise tant à l'interne qu'à l'externe représente une valeur qui nous définit depuis les premières années de notre existence. II ne saurait donc être question de l'abandonner maintenant. 


\section{6. ÉVALUER ET MESURER NOS SERVICES}

Former et informer la communauté universitaire à nos produits et services a toujours été et demeure une préoccupation de chaque instant. Mais après 50 ans d'existence, quel est notre réel degré de notoriété, que connaît-on de nous et quelle est la perception de notre mandat et de notre performance dans les unités? Difficile de le dire sans un coup de sonde chez nos partenaires.

Alors que le RAQ travaille avec I'AAQ et BAnQ, à la préparation d'une enquête statistique sur les services d'archives, il serait intéressant d'aller voir sur le terrain comment nous sommes perçus afin d'évaluer notre performance et d'identifier les points à améliorer pour répondre aux besoins des utilisateurs.

Un tel exercice devrait être entrepris à court terme et réédité de temps à autre afin de s'assurer que nous sommes toujours sur la bonne voie. C'est là un autre défi qui nous attend et auquel nous devrons nous attaquer prochainement.

Beaucoup a été accompli au cours des 50 dernières années, mais il reste encore tellement à faire. Les défis sont importants, mais avec une équipe compétente, enthousiaste et bien préparée à les relever rien ne saurait nous arrêter.

\section{BIBLIOGRAPHIE}

MAUREL, D. (2013). Gouvernance informationnelle et perspective stratégique. Dans Clavier V. et C. Pagnelli (dir.). L'information professionnelle. Paris: Lavoisier.

UNIVERSITÉ DE MONTRÉAL. DIVISION DE LA GESTION DE DOCUMENTS ET DES ARCHIVES. (2016). Mission, vision, valeurs. Repéré à http://www. archiv.umontreal.ca/information/mission_vision_valeurs Web.pdf. 